\title{
Caracterização Química e Estrutural de Fibra de Sisal da Variedade Agave sisalana
}

\author{
Adriana R. Martin \\ Departamento de Engenharia de Materiais, UFSCar \\ Maria A. Martins, Luiz H. C. Mattoso \\ Embrapa Instrumentação Agropecuária, São Carlos/SP \\ Odilon R. R. F. Silva \\ Embrapa Algodão, Campina Grande/PB
}

Resumo: Nos últimos anos, o interesse pelo uso de fibras naturais em materiais compósitos poliméricos tem aumentado significativamente. Neste trabalho foram investigadas as propriedades, químicas, físicas, térmicas e estruturais da fibra de sisal brasileira da variedade Agave sisalana. Nosso objetivo foi avaliar a qualidade e o desempenho desta fibra para aplicações industriais. Foram realizados ensaios de resistência à tração, análise da composição química, difração de raios X, e estudos por microscopia eletrônica de varredura (MEV) ao longo do comprimento da fibra. A fibra de sisal brasileira apresentou propriedades mecânicas e térmicas dentro da faixa relatada na literatura, mostrando-se adequada para ser utilizada em materiais compósitos poliméricos.

Palavras-chave: Fibras naturais, compósitos, composição química, caracterização.

\section{Chemical and Structural Characterization of Sisal Fibers from Agave sisalana Variety}

\begin{abstract}
In recent years, the interest in the use of natural fibers in polymeric composite materials has increased significantly. In this work were investigated the structural, thermal, chemical, and physical properties of Brazilian sisal fiber from Agave sisalana variety. Our aim was to evaluate the quality and the performance of this fiber for industrial applications. Mechanical properties, chemical composition, X ray diffraction, and scanning electron microscopy (SEM) have been investigated with fibers along their length. The Brazilian sisal fibers studied have exhibited mechanical and thermal properties within the range reported in the literature and were suitable for use in polymeric composites.
\end{abstract}

Keywords: Natural fibers, composites, chemical composition, characterization.

\section{Introdução}

O sisal é a principal fibra dura produzida no mundo, correspondendo a aproximadamente $70 \%$ da produção comercial de todas as fibras desse tipo. No Brasil, o cultivo do sisal se concentra na região Nordeste, sendo os estados da Bahia, Paraíba e Rio Grande do Norte os principais produtores, com 93,5, 3,5 e 3,0\%, respectivamente, da produção nacional ${ }^{[1-3]}$. A agaveicultura se concentra em áreas de pequenos produtores, com predomínio do trabalho familiar. O sisal, além de constituir fonte de renda e emprego para um grande contingente de trabalhadores, é um importante agente de fixação do homem à região semi-árida nordestina, sendo, em algumas dessas regiões, a única alternativa de cultivo com resultados econômicos satisfatórios. A fibra do sisal, beneficiada ou industrializada, representa cerca de 80 milhões de dólares em divisas para o Brasil, além de gerar mais de meio milhão de empregos diretos e indiretos por meio de sua cadeia de serviços, que começa com as atividades de manutenção das lavouras, colheita, desfibramento e beneficiamento da fibra e termina com a industrialização e confecção de $\operatorname{artesanato~}^{[1,2,4-6]}$.

A planta do sisal é originária da península de Yucatã, no México, tendo recebido este nome de uma erva nativa chamada zizal-xiu ${ }^{[4]}$. Em 1834, as primeiras mudas foram levadas para o sul da Flórida (EUA) e a partir de 1892 começaram a ser cultivadas na África. No Brasil, as primeiras mudas de Agave sisalana Perrine foram introduzidas na Bahia em 1903, sendo esta a única espécie do gênero Agave cultivada comercialmente no país ${ }^{[5-7]}$. As fibras são extraídas das folhas, que possuem de 8 a $10 \mathrm{~cm}$ de largura e de 150 a $200 \mathrm{~cm}$ de comprimento. Da folha se obtém de 3 a 5\% do seu peso em fibra. Os 95 a 97\% restantes constituem os chamados resíduos do beneficiamento, que são utilizados como adubo orgânico, ração animal e pela indústria farmacêutica. As fibras são constituídas de fibrilas ou

Autor para correspondência: Luiz H. C. Mattoso, Embrapa Instrumentação Agropecuária, Rua XV de Novembro 1452, Caixa Postal 741, CEP: 13560-970, São Carlos, SP, Brasil. E-mail: mattoso@cnpdia.embrapa.br 
fibras elementares que tem de 1,5 a $4 \mathrm{~mm}$ de comprimento, diâmetro de 10 a $30 \mu \mathrm{m}$, e a espessura da parede celular varia de 6 a $9 \mu \mathrm{m}$. A parede celular é constituída de várias camadas, que diferem umas das outras em relação à sua estrutura e composição química ${ }^{[1,3,6-8]}$.

Atualmente, o maior produtor e exportador mundial de fibras de sisal e de sisal manufaturado é o Brasil ${ }^{[6,8]}$, e aproximadamente $70 \%$ do sisal brasileiro beneficiado destina-se aos mercados europeu e asiático. Já o sisal manufaturado tem como principais importadores os Estados Unidos (86\%) e o Canadá (5\%). A produção nacional de fibras de sisal já alcançou 135 mil toneladas, destas, 113 mil foram exportadas, sendo $26 \%$ de fibras e $74 \%$ de manufaturados ${ }^{[2,8]}$. As principais aplicações da fibra de sisal industrializada são na indústria automobilística e também na fabricação de cordas, barbante, cabos marítimos, tapetes, sacos, vassouras, estofamentos, e artesanato; além disso, tem utilização industrial na fabricação de pasta celulósica para produção do papel Kraft de alta resistência, e de outros tipos de papel fino, como para cigarro, filtro, absorvente higiênico, fralda, etc ${ }^{[2,8,11]}$.

Além destas aplicações, a literatura relata diversos estudos sobre a modificação da superfície das fibras com objetivo de sua utilização em materiais compósitos poliméricos de grande interesse para a indústria ${ }^{[1,3,7,8,10,12-20]}$. Os compósitos reforçados com fibras de sisal se destacam por apresentarem alta resistência ao impacto, e boas propriedades de resistência à tração e flexão ${ }^{[3,21-24]}$. Isto é atribuído ao fato da fibra de sisal apresentar um dos maiores valores de módulo de elasticidade e de resistência mecânica entre as fibras naturais ${ }^{[25]}$.

Nos últimos anos, o interesse da indústria pelo uso de fibras naturais para o reforço de plásticos e a obtenção de materiais com desempenho mecânico e térmico adequado, tem aumentado significativamente ${ }^{[3,7,8,26]}$. A utilização de fibras naturais em substituição às fibras sintéticas como reforço de compósitos poliméricos é uma possibilidade bastante atraente, principalmente por ser biodegradável, atóxica, de fonte renovável e apresentar baixo custo, o que condiz com os atuais esforços de proteção ao meio ambiente ${ }^{[3,7,8,21,22]}$. As fibras lignocelulósicas provocam pouco dano aos equipamentos durante os métodos convencionais de processamento de polímeros, quando comparadas às fibras inorgânicas (fibras de vibro e asbesto, por exemplo), e possuem também menor densidade ${ }^{[4,5]}$

Embora na literatura, se encontrem vários estudos de compósitos poliméricos com fibras de sisal ${ }^{[1,3,6,9,12-15,21,22,24-27]}$, pouco se tem desenvolvido na caracterização da fibra, e em particular no que diz respeito a análises físico-químicas e morfológicas visando um maior entendimento de suas propriedades. O estudo para a determinação da composição química da fibra, realizado por Medina ${ }^{[5]}$ mostrou que a fibra é constituída de $65,8 \%$ de celulose, $12 \%$ de hemicelulose, $9,9 \%$ de lignina e $0,8 \%$ de pectina. No trabalho realizado por Mwaikambo e colaboradores ${ }^{[9]}$, os resultados obtidos foram $73 \%$ de celulose, $13 \%$ de hemicelulose, $11 \%$ de lignina e $2 \%$ de pectina. Para o índice de cristalinidade, o valor determi- nado por Ferreira e colaboradores ${ }^{[10]}$ foi de $75 \%$ e o obtido por Mwaikambo e colaboradores ${ }^{[9]}$ foi igual a $70,9 \%$. Desta forma, este trabalho teve como objetivo o estudo das propriedades físicas e estruturais da fibra de sisal brasileira da variedade Agave sisalana, ao longo do comprimento da fibra, o que é essencial para várias aplicações tecnológicas deste material.

\section{Experimental}

As amostras da fibra de sisal foram fornecidas pela Embrapa/CNPA de Campina Grande/PB. As fibras utilizadas foram da variedade Agave sisalana, de 4 anos de idade e do segundo ano de colheita. A caracterização desta variedade de sisal foi realizada ao longo do comprimento da fibra na folha, que foi cortada em 4 partes iguais, referentes às faixas de comprimentos: 0-30 cm (denominada basal), 30-60, $60-90$ e $90-120 \mathrm{~cm}$ (denominada apical). Foram realizados ensaios de resistência à tração, análise da composição química, difração de raios $\mathrm{X}$, análise térmica, e estudos por microscopia eletrônica de varredura (MEV).

A composição química da fibra foi determinada de acordo com os métodos descritos por Browning ${ }^{[28]}$, e Rowell ${ }^{[29]}$. Inicialmente, as fibras foram moídas em um moinho de facas e passadas em uma peneira de 16 mesh. Em seguida, foram submetidas à extração com ciclohexano/etanol 1:1, por 48 horas, para remover os extrativos solúveis em solventes orgânicos e, em seguida, mais 6 horas em água para remoção dos solúveis em água. Foram secas em estufa a $105^{\circ} \mathrm{C} \pm 5{ }^{\circ} \mathrm{C}$ por 4 horas até atingir massa constante. Após estes procedimentos, foram realizadas as análises, em triplicata, para determinação do teor de lignina, hemicelulose, celulose e cinzas.

As medidas de difração de raios $\mathrm{X}$ foram realizadas em um difratômetro Siemens, nas condições de $40 \mathrm{kV}$ e $30 \mathrm{~mA}$. $\mathrm{O}$ índice de cristalinidade (Ic) da celulose foi calculado através da equação: $\mathrm{Ic}=1-\mathrm{I}_{1} / \mathrm{I}_{2}{ }^{[7,30,31]}$, onde $\mathrm{I}_{1}=$ intensidade do mínimo de difração, relacionada à parte amorfa $\left(2 \theta=18,8^{\circ}\right)$ e $\mathrm{I}_{2}=$ intensidade do máximo de difração, relacionada à parte cristalina $\left(2 \theta=22,4^{\circ}\right)$. Este método foi desenvolvido por Segal e colaboradore ${ }^{[30]} \mathrm{e}$ tem sido largamente utilizado para o estudo de fibras naturais ${ }^{[7,9,10,31-35]}$.

Os ensaios de tração das fibras foram realizados em uma Máquina Universal de Ensaio Instron 4442, com velocidade de deslocamento das garras de $30 \mathrm{~mm} / \mathrm{min}$, e célula de carga de $500 \mathrm{~N}$, em fibras com $30 \mathrm{~cm}$ de comprimento. As condições dos ensaios foram baseadas nas normas ASTM D 2256-95a $\mathrm{a}^{[36]}$ e ASTM D 76-90 $0^{[37]}$, foram ensaiadas pelo menos 50 fibras para cada amostra.

O diâmetro das fibras foi calculado a partir do valor médio da medida de densidade obtida por picnometria de hélio. Para este cálculo, foram utilizadas amostras com comprimento fixo de $30 \mathrm{~cm}$ e assumiu-se que a fibra possui secção transversal circular.

A termogravimetria (TG/DTG) foi realizada em um equipamento Shimadzu, com fluxo de nitrogênio de $20 \mathrm{~mL} / \mathrm{min}$, 
razão de aquecimento de $10{ }^{\circ} \mathrm{C} / \mathrm{min}$, e faixa de temperatura da ambiente até $600{ }^{\circ} \mathrm{C}$.

Os ensaios por DSC foram feitos em um equipamento Shimadzu, modelo DSC 50, com fluxo de nitrogênio de $20 \mathrm{~mL} / \mathrm{min}$, razão de aquecimento de $10{ }^{\circ} \mathrm{C} / \mathrm{min}$, e faixa de temperatura da ambiente a $600{ }^{\circ} \mathrm{C}$.

A caracterização através de microscopia eletrônica de varredura (MEV) foi realizada em um microscópio Zeiss DSM 960, operando a $20 \mathrm{kV}$ na modalidade de SEI (elétrons secundários). Após a preparação das amostras, foi feita a deposição de ouro pelo método de "Sputtering", utilizando um equipamento Sputter Coater Balzers SCD 50.

\section{Resultados e Discussão}

As fibras celulósicas apresentam ordenamento tridimensional com diferentes graus de perfeição. Em um modelo simplificado de duas fases, a estrutura supramolecular de um polímero é considerada como um sistema de fases cristalinas (ordenada) e amorfas (desordenada) ${ }^{[7,31,32]}$. Segundo Sao et al. ${ }^{[38]}$, as propriedades mecânicas dos materiais lignocelulósicos são fortemente dependentes do índice de cristalinidade dos mesmos. Nas últimas décadas, a medida do índice de cristalinidade tem recebido muita atenção por razões de aplicações comerciais e devido à importância que estes materiais vêm despertando na área industrial ${ }^{[39]}$. A composição química das fibras, a variação do índice de cristalinidade (Ic) $\mathrm{e}$ as curvas de raios $\mathrm{X}$ ao longo do comprimento da fibra de sisal são apresentadas na Tabela 1 e na Figura 1, respectivamente. Observa-se que o Ic apresenta comportamento similar ao apresentado pela variação da porcentagem de celulose, aumentando ao longo da fibra da parte basal (1) para a apical (4) à medida que aumenta o conteúdo de celulose presente nas fibras e diminui os conteúdos de hemicelulose e lignina.

Hu et al. ${ }^{[40]}$ realizando estudos com celulose proveniente de algodão observou que a reflexão no plano (002) corresponde aos planos de rede dos anéis glicosídicos, que são os mais densos na estrutura da celulose do tipo I. Segundo a literatura ${ }^{[41-43]}$ a forma polimórfica da celulose que ocorre naturalmente é apenas a do tipo I. Também de acordo com Hu et al..$^{[40]}$, a intensidade do pico da reflexão 002 aumenta, e sua largura diminui com o desenvolvimento da fibra, indicando que ocorre um aumento no alinhamento dos anéis glicosídicos no plano (002) com o desenvolvimento da biosíntese do algodão, ocorrendo um aumento na porção cristalina da mesma.

Apesar das interferências das regiões amorfas da celulose, pode-se observar, Figura 1, a presença dos picos refe-

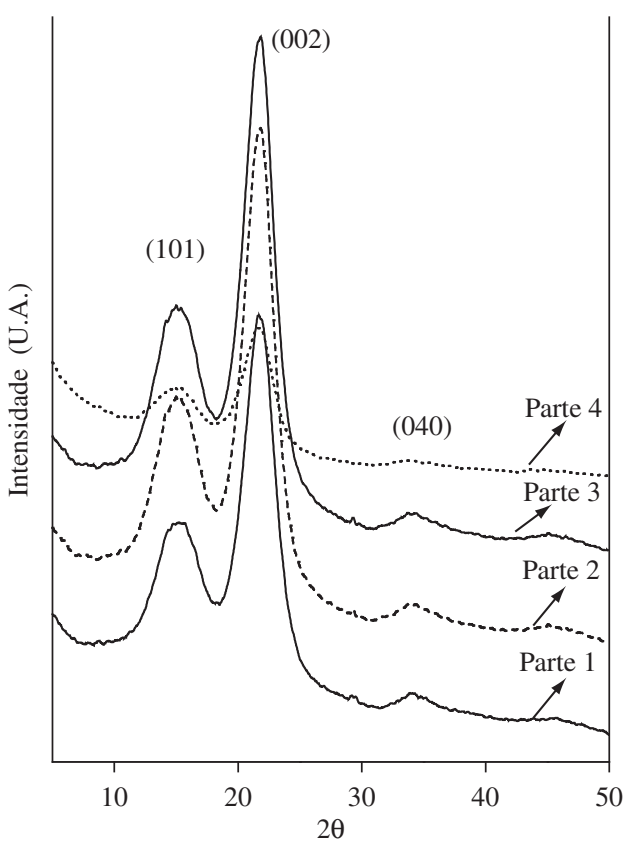

Figura 1. Difratogramas de raio $X$ ao longo do comprimento da fibra de sisal da variedade Agave sisalana. Parte 1: 0-30 cm (parte basal); parte 2: 30-60 cm; parte 3: 60-90 cm; e parte 4: 90-120 cm (parte apical).

rentes aos planos cristalográficos nos seguintes ângulos de

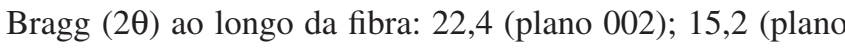
101) e 34,4 (plano 040). Os resultados de raios $X$ apresentam os picos referentes aos planos cristalinos característicos dos materiais lignocelulósicos, onde se pode observar que a reflexão (002) é a mais intensa em todas as partes ao longo da fibra. A fibra de sisal é constituída basicamente de lignina, hemicelulose e celulose, sendo que a lignina e a hemicelulose são macromoléculas amorfas, e as moléculas de celulose são orientadas aleatoriamente tendo a tendência de formar ligações de hidrogênio intra e intermoleculares. Assim, à medida que a densidade de empacotamento da celulose aumenta, regiões cristalinas são formadas ${ }^{[29]}$. Portanto, pode-se dizer que o aumento do índice de cristalinidade refere-se ao aumento da quantidade de celulose ao longo da fibra como foi observado nas análises de composição química, Tabela 1.

Rowell et al. ${ }^{[29,44]}$ demonstraram que a composição química de fibras lignocelulósicas influencia as suas propriedades mecânicas e estrutura física, sendo que o conteúdo de lignina e hemicelulose presente em fibras como a de sisal, tem efeito sobre a resistência à tração destas fibras ${ }^{[45]}$. De acordo com Bisanda et al. ${ }^{[46]}$, a resistência e a rigidez das fibras dependem do conteúdo de celulose e do ângulo espiral que

Tabela 1. Composição química média e índice de cristalinidade (Ic) ao longo do comprimento da fibra de sisal da variedade Agave sisalana. Triplicata de amostra.

\begin{tabular}{ccccccc}
\hline Parte & Posição na fibra & Lignina (\%) & Hemicelulose (\%) & Celulose (\%) & Cinzas (\%) & Ic \\
\hline 1 & $0-30 \mathrm{~cm}$ & $11,4 \pm 0,6$ & $10,3 \pm 0,3$ & $77,3 \pm 0,1$ & 1,5 & 0,81 \\
2 & $30-60 \mathrm{~cm}$ & $10,7 \pm 0,8$ & $9,9 \pm 0,5$ & $79,6 \pm 0,2$ & 1,5 & 0,84 \\
3 & $60-90 \mathrm{~cm}$ & $8,1 \pm 0,8$ & $8,0 \pm 0,2$ & $83,0 \pm 0,9$ & 0,8 & 0,85 \\
4 & $90-120 \mathrm{~cm}$ & $7,4 \pm 0,4$ & $6,9 \pm 0,3$ & $84,4 \pm 0,8$ & 1,2 & 0,91 \\
\hline
\end{tabular}


as microfibrilas da parede celular secundária interna faz com o eixo da fibra. A composição química média ao longo do comprimento das fibras é apresentada na Tabela 1, onde se pode observar que ocorre diminuição do conteúdo de lignina e hemicelulose da parte basal (parte 1) para a apical (parte 4) em cerca de 35 e $33 \%$, respectivamente. Observa-se também que o conteúdo de celulose aumenta cerca de $10 \%$ da parte 1 para a 4 , e a porcentagem de cinzas varia ao longo da fibra, mostrando uma tendência à diminuição da parte basal para a apical. Segundo Rowell et al. ${ }^{[29]}$ a maioria da celulose derivada de plantas é altamente cristalina e pode apresentar mais do que $80 \%$ de regiões cristalinas, resultado que concorda com os valores de celulose obtidos para as fibras de sisal estudadas, que variou de cerca de 77 a 84\%, Tabela 1.

Os resultados dos ensaios das propriedades mecânicas e do diâmetro ao longo do comprimento da fibra de sisal são apresentados na Tabela 2 . A resistência à tração aumenta de 448 para $671 \mathrm{MPa}$ da parte $1(0-30 \mathrm{~cm})$ para a $2(30-60 \mathrm{~cm}) \mathrm{e}$, depois cai para $565 \mathrm{MPa}$ na parte $3(60-90 \mathrm{~cm})$ e para $369 \mathrm{MPa}$ na parte $4(90-120 \mathrm{~cm})$. O módulo de elasticidade apresentou a mesma tendência, ou seja, aumentou de $10 \mathrm{GPa}$ para 25 GPa da parte 1 para a 2 e depois decresceu de 22 (parte 3 ) para $17 \mathrm{GPa}$, na parte 4 . Com relação à porcentagem de alongamento, observou-se uma queda ao longo do comprimento da fibra. Este decréscimo na porcentagem de alongamento pode estar associado ao aumento da porcentagem de celulose ao longo do comprimento. Os resultados da medida do diâmetro da fibra mostraram que este diminui em cerca de um terço da parte basal para a apical. Da parte 1 para as partes 2 e 3 , ocorreu uma diminuição de cerca de $5 \%$ e das partes 2 e 3 para a parte 4 , a diminuição do valor do diâmetro é de aproximadamente $65 \%$. Este acentuado decréscimo da parte basal para a apical explica a grande variação dos valores de diâmetro para a fibra de sisal relatados na literatura ${ }^{[3,8,11,23]}$.

Os resultados obtidos para a fibra de sisal desta variedade cultivada no Brasil em relação às propriedades de resistência à tração, módulo de elasticidade e porcentagem de alongamento mostraram que a resistência e o módulo de elasticidade na região da folha próxima à raiz ou parte basal são baixos, enquanto que a deformação é alta, sendo a parte intermediária mais resistente e rígida e, a ponta ou parte apical apresenta resistência e rigidez moderada ${ }^{[47,48]}$. Portanto, os resultados das propriedades mecânicas ao longo do comprimento para a fibra brasileira indicam que estas possuem propriedades satisfatórias para o reforço de compósitos poliméricos e/ou em outras aplicações industriais, sendo as melhores propriedades obtidas na parte $2(30-60 \mathrm{~cm})$ e o menor desempenho na parte apical $(90-120 \mathrm{~cm})$. Os altos valores obtidos para os desvios padrão das propriedades mecânicas estão, em geral, correlacionados com a variação de diâmetro e com parâmetros estruturais das fibras naturais.

Segundo a literatura ${ }^{[49,50]}$, a variação da resistência à tração e o módulo de elasticidade estão relacionados diretamente com a variação do índice de cristalinidade (Ic), ou seja, com a quantidade de celulose presente nas fibras uma vez que a celulose é o único componente presente na fibra que se cristaliza $^{[29]}$. Entretanto, os resultados obtidos mostram que não há uma correlação direta entre as propriedades de resistência à tração, o módulo e o conteúdo de celulose ao longo da fibra de sisal. Estudos realizados para correlacionar a estrutura e as propriedades mecânicas em fibras naturais têm demonstrado que o desempenho mecânico das fibras naturais depende também de outros parâmetros estruturais como o ângulo ou orientação das microfibrilas e cristais de celulose que compõem a fibra, e do número, comprimento, e largura das células unitárias ${ }^{[32,51,52]}$.

A caracterização térmica da fibra de sisal é apresentada nas Figuras 2 e 3. Foram realizados ensaios de DSC e TG ao longo do comprimento da fibra, sendo que os resultados obtidos não apresentaram diferença significativa. As Figuras 2 e 3 mostram as curvas obtidas por amostragem aleatória das diferentes partes da fibra, são apresentadas as curvas representativas dos resultados obtidos. Na Figura 2 são apresentadas as curvas termogravimétricas (TG/DTG) da fibra de sisal e observa-se, que ocorreu cerca de $3 \%$ de perda de massa na faixa de temperatura de 30 a $220{ }^{\circ} \mathrm{C}$, atribuída à perda da água presente nas fibras (reações de desidratação intra e intermoleculares $)^{[53]}$. A degradação da fibra de sisal inicia-se em aproximadamente $250{ }^{\circ} \mathrm{C}$ e continua rapidamente com o aumento da temperatura, apresentando perda de massa de

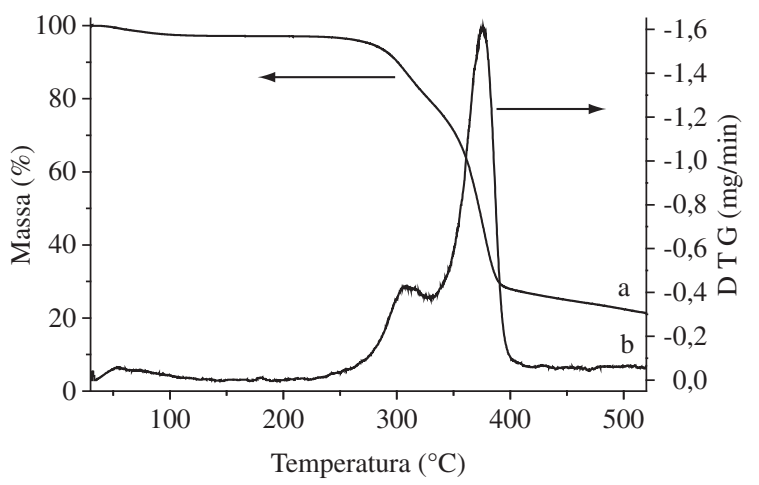

Figura 2. Curvas TG/DTG da fibra de sisal da variedade Agave sisalana. Atmosfera de nitrogênio, razão de aquecimento de $10^{\circ} \mathrm{C} / \mathrm{min}$.

Tabela 2. Propriedades mecânicas ao longo do comprimento da fibra de sisal da variedade Agave sisalana. Foram ensaiadas pelo menos 50 fibras para cada amostra.

\begin{tabular}{cccccc}
\hline Parte & Posição na fibra & Resistência à tração $(\mathbf{M P a})$ & Módulo elástico $(\mathbf{G P a})$ & Alongamento $(\%)$ & Diâmetro $(\mu \mathbf{m})$ \\
\hline 1 & $0-30 \mathrm{~cm}$ & $448 \pm 113$ & $10 \pm 3$ & $5 \pm 1$ & $465 \pm 20$ \\
2 & $30-60 \mathrm{~cm}$ & $671 \pm 274$ & $25 \pm 4$ & $3 \pm 1$ & $430 \pm 9$ \\
3 & $60-90 \mathrm{~cm}$ & $565 \pm 146$ & $22 \pm 4$ & $3 \pm 1$ & $440 \pm 20$ \\
4 & $90-120 \mathrm{~cm}$ & $369 \pm 129$ & $17 \pm 3$ & $2 \pm 1$ & $143 \pm 14$ \\
\hline
\end{tabular}




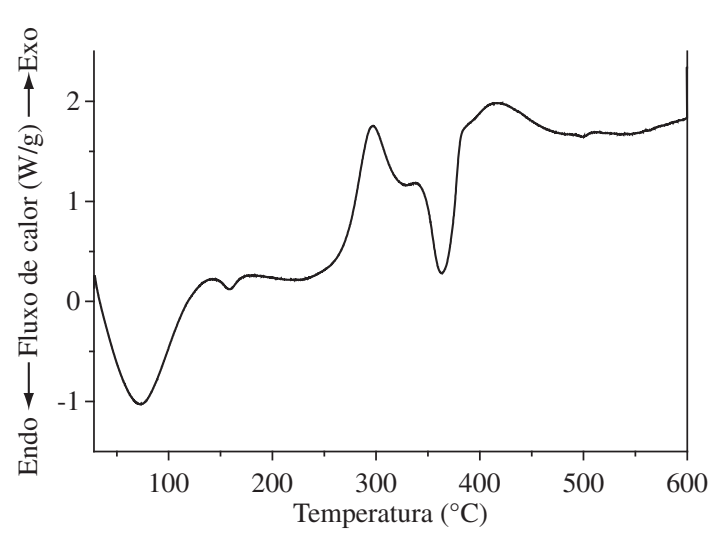

Figura 3. Curva DSC da fibra de sisal da variedade Agave sisalana. Atmosfera de nitrogênio, razão de aquecimento de $10{ }^{\circ} \mathrm{C} / \mathrm{min}$.

$72 \%$ até cerca de $420{ }^{\circ} \mathrm{C}$. Devido à lenta taxa de decomposição dos resíduos, a perda de massa a partir de $420{ }^{\circ} \mathrm{C}$ até cerca de $520^{\circ} \mathrm{C}$ é apenas cerca de $5 \%$, e a $520^{\circ} \mathrm{C}$ o teor de cinzas é de aproximadamente $20 \%$ devido à condensação dos componentes da lignina e de sua aromatização em atmosfera de nitrogênio a altas temperaturas, além da presença do conteúdo de cinzas na fibra. A curva DTG, Figura 2, apresentou dois picos distintos com máximos em cerca de 310 e $375^{\circ} \mathrm{C}$, indicando que ocorrem duas etapas na degradação térmica da fibra. O primeiro pico é atribuído à decomposição de componentes da hemicelulose, e observa-se que na faixa de temperatura de 220 a $330^{\circ} \mathrm{C}$ ocorre degradação lenta com perda de massa de $18 \%$. A degradação da celulose ocorre em temperaturas mais altas em relação a hemicelulose e também a taxas mais rápida de decomposição, o principal pico inicia-se em $330{ }^{\circ} \mathrm{C}$ e finaliza em cerca de $420^{\circ} \mathrm{C}$ sendo que seu máximo ocorre na temperatura de $375^{\circ} \mathrm{C}^{[7]}$.

Uma curva de DSC representativa da fibra de sisal, em atmosfera de nitrogênio, é apresentada na Figura 3, observamse os picos referentes aos três maiores constituintes da fibra, celulose, hemicelulose e lignina. A endoterma observada em aproximadamente $75^{\circ} \mathrm{C}$ é atribuída à evaporação da umidade presente nas amostras. $\mathrm{O}$ pico exotérmico observado na curva térmica da fibra de sisal iniciando em $234^{\circ} \mathrm{C}$, com máximo em $297{ }^{\circ} \mathrm{C}$ é devido à degradação da fração de hemicelulose, ou seja, do acetil 4-O-metilglucuronoxilano do sisal ${ }^{[54]}$. E a endoterma iniciando em $328{ }^{\circ} \mathrm{C}$ com mínimo em $364{ }^{\circ} \mathrm{C}$ é atribuída à degradação da celulose, que é o constituinte predominante da fibra ${ }^{[7,8]}$. Bhaduri et al. ${ }^{[54]}$ e Shafizadeh et al. ${ }^{[5]}$ observaram que esta endoterma é conseqüência da desidratação e da despolimerização do componente celulose da fibra, levando à formação de produtos voláteis flamáveis. Chand et al ${ }^{[56]}$ atribuem esta endoterma a desidratação e à quebra de grupos hidroxila da molécula de celulose, resultando na evolução de água. Em seu trabalho, Fairbridge et al..$^{[53]}$ realizaram estudos de decomposição de celulose em atmosferas de nitrogênio e oxigênio e observaram que a degradação da celulose ocorre via duas reações competitivas de primeira ordem, as quais produzem uma fração condensada, uma fração gasosa e cinzas.

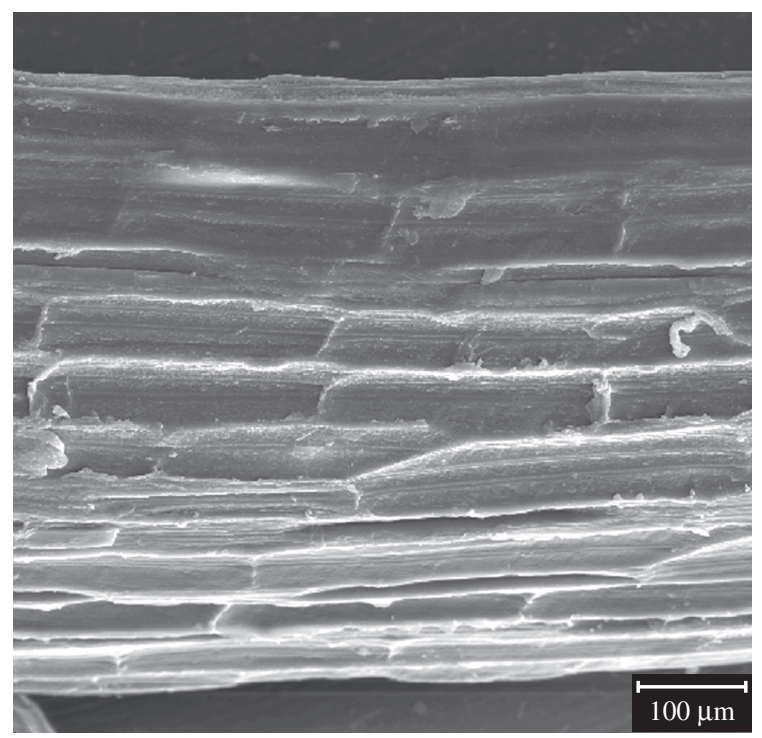

(a)

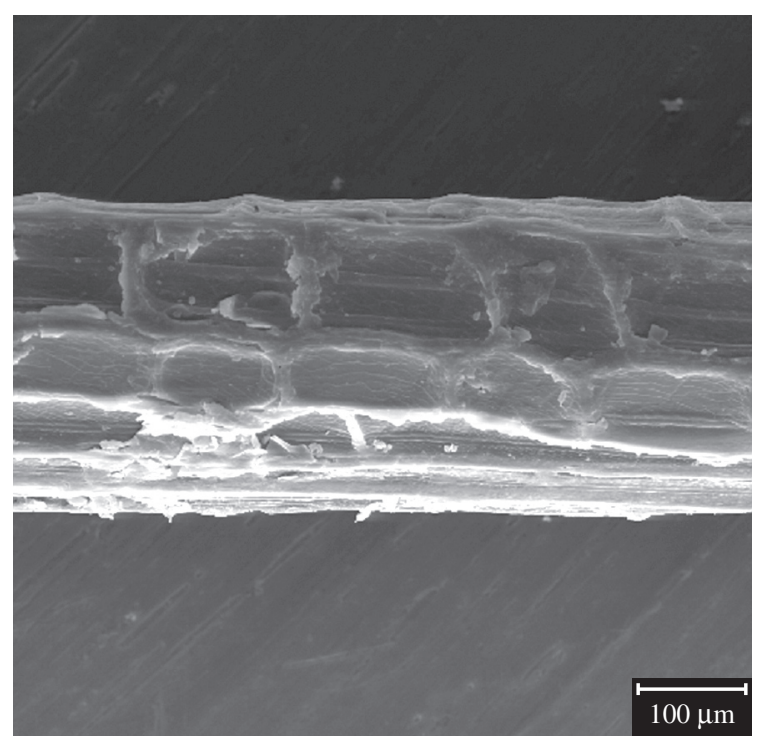

(b)

Figura 4. a) Micrografias obtidas por MEV da parte basal $(0-30 \mathrm{~cm})$; e b) parte apical $(90-120 \mathrm{~cm})$ da fibra de sisal da variedade Agave sisalana. 150x.

Micrografias representativas obtidas por MEV das partes basal e apical da superfície longitudinal da fibra de sisal são apresentadas na Figura 4. Pode-se verificar a variação do diâmetro ao longo da fibra, que diminui da parte basal para a apical, concordando com os dados mostrados na Tabela 2 . Observam-se ainda, as células do parênquima largamente distribuídas ao longo de toda a fibra. As partes 2 e 3 da fibras apresentaram características morfológicas e diâmetro semelhante a parte 1 . De acordo com a literatura ${ }^{[57]}$, as fibras são formadas por um feixe composto de células individuais denominadas fibras elementares ou microfibrilas, unidas entre si pela lignina e polioses, de maneira a formar filamentos contínuos em todo o sentido do comprimento da fibra e proporcionando, desta forma, uma certa rigidez a mesma. 


\section{Conclusões}

A fibra de sisal brasileira da variedade Agave sisalana apresentou propriedades mecânicas dentro da faixa relatada na literatura, mostrando-se adequada para ser utilizada em materiais compósitos poliméricos, assim como em outras aplicações. As fibras apresentaram, também, boa estabilidade térmica até cerca de $250^{\circ} \mathrm{C}$, podendo desta forma, ser utilizadas para processamento com a maioria dos polímeros na produção de compósitos. Em relação às propriedades ao longo do comprimento da fibra observou-se que a parte mais resistente da fibra está localizada cerca de 30 a $60 \mathrm{~cm}$ da sua base e a de menor desempenho na região apical (90-120 cm).

\section{Agradecimentos}

Os autores agradecem à FAPESP, ao CNPq, à FINEP e à FULBRIGHT (EUA) pelo suporte financeiro.

\section{Referências Bibliográficas}

1. Mattoso, L. H. C.; Ferreira, F. C. \& Curvelo, A. A. S. - "Lignocellulose-Plastic Composites", Leão, A. L.; Carvalho, F. X.; Frollini, E. (ed.), USP \& UNESP, São Paulo (1997).

2. http://www.cnpa.embrapa.br - acessado em Maio/2007.

3. Li, Y.; Mai; Y. W. \& Ye, L. - Composites Sci. Technol., 6, p.2037 (2000).

4. Sisal, Informe Técnico, Centro de Planejamento da Bahia: Salvador (1980).

5. Medina, J. C. - "O sisal”, Secretaria da Agricultura do Estado de São Paulo, São Paulo (1954).

6. Silva, O. R. R. \& Beltrão, N. R. R. F. - "O Agronegócio do Sisal no Brasil”, Embrapa, Brasília (1999).

7. Martin, A. R. - "Caracterização e Modificação de Fibras de Sisal por Plasma a Frio Visando Aplicação em Compósitos Poliméricos”. Tese de Doutorado, Universidade Federal de São Carlos, Brasil (2001).

8. Martins, M. A. - "Fibra de Sisal: Mercerização, Acetilação e Aplicação em Compósitos de Borracha de Pneu Triturado", Tese de Doutorado, Universidade Estadual de Campinas, Brasil (2001).

9. Mwaikambo, L.Y. \& Ansell, M. P. - J. Appl. Polym. Sci., 84, p.2222 (2002).

10. Ferreira, F. C.; Curvelo, A. A. S. \& Mattoso, L. H. C. - J. Appl. Polym. Sci., 89, p.2957 (2003).

11. Mukherjee, P. S. \& Satyanarayana, K. G. - J. Mater. Sci., 19, p.3925 (1984).

12. Martin, A. R.; Manolache, S.; Denes, F. S. \& Mattoso, L. H. C. - J. Appl. Polym. Sci., 85, p.2145 (2002).

13. Li, X.; Tabil, L. G. \& Panigrahi, S. - J. Polym. Environ., 15, p.25 (2007).
14. Mwaikambo, L.Y. \& Ansell, M. P. - Die Angewandre Makromolekulare Chemie, 272, p.108 (1999).

15. Martins, M. A. \& Joekes, I. - J. Appl. Polym. Sci., 89, p.2507 (2003).

16. Pinto, M. R. O.; Júnior, M. M. A.; Silva, S. M. I. \& de Carvalho, L. H. - Polímeros, 15, p.313 (2005).

17. Fonseca, V. M.; Fernandes Jr., V. J.; de Carvalho, L. H. \& d'Almeida, J. R. M. - J. Appl. Polym. Sci., 94, p.1209 (2004).

18. Alsina, O. L. S.; de Carvalho, L. H.; Ramos Filho, R. G. \& d'Almeida, J. R. M. - Polym. Testing, 24, p.81 (2005).

19. d'Almeida, A. L. F. S.; Calado, V.; Barreto, D. W. \& d'Almeida, J. R. M. - Polímeros, 15, p.59 (2005).

20. Costa, F. H. M. M. - Polym.-Plast. Technol. Eng., 38, p.1081 (1999).

21. Martin, A. R.; Denes, F. S.; Rowell, R. M. \& Mattoso, L. H. C. - Polym. Composites, 24, p.464 (2003).

22. Martins, M. A. \& Mattoso, L. H. C. - J. Appl. Polym. Sci., 91, p.670 (2004).

23. Mishra, S.; Mohanty, A. K.; Drzal, L. T.; Misra, M. \& Hinrichsen, G. - Macromol. Mater. Eng., 289, p.955 (2004).

24. Joseph, K.; Thomas, S. \& Pavithran, C. - Polymer, 37, p.5139 (1996).

25. Bledzki, A. K. \& Gassan, J. - Progress Polym. Sci., 24, p.221 (1999).

26. Joseph, P. V.; Joseph, K. \& Thomas, S. - Composites Sci. Technol., 59, p.1625 (1999).

27. Bisanda, E. T. N. \& Ansell, M. P. - Composites Sci. Technol., 41, p.165 (1991).

28. Browning, B. L. - "Methods of Wood Chemistry", John Wiley \& Sons, New York (1967).

29. Rowell, R. M.; Young, R. A. \& Rowell, J. K. - "Paper and Composites from Agro-Based Resources", Lewis Publishers, New York (1997).

30. Segal, L.; Creely, J. J.; Martin, A. E. \& Conrad, C. M. Textile Res. J., 29, p.786 (1959).

31. Maldas, D. \& Kokta, B. V. - J. Adhes. Sci. Technol., 5, p.727 (1991).

32. Tomaz, R. M. A. G.; Bittencourt, E.; Sabino, N. P. \& Kondo, J. I. - Bragantia, 53, 121 (1994).

33. Wang, B.; Sain, M. \& Oksman, K. - Appl. Compos. Mater., 14, p.89 (2007).

34. Meunier-Goddik, L.; Bothwell, M.; Sangseethong,K.; Piyachomkwan, K.; Chung, Y.; Thammasouk, K.; Tanjo, D. \& Penner, M. H. - Enzyme Microbial Technol., 24, p.667 (1999).

35. Ass, B. A. P.; Belgacemb, M. N. \& Frollini, E. - Carbohyd. Polym., 63, p.19 (2006). 
36. American Society for Testing and Materials. Standard Test Method for Tensile Properties of Yarns by SingleStrand Method. ASTM D 2256a (1990).

37. American Society for Testing and Materials. Standard Specification Tensile Testing Machines for Textiles. ASTM D 76 (1990).

38. Sao, K. P.; Samantaray, B. K. \& Bhattacherjee, S. - J. Appl. Polym. Sci., 52, p.1687 (1994).

39. Moharir, A.V. \& Kiekens, P. - J. Appl. Polym. Sci., 68, p.2107 (1998).

40. Hu, X. \& Hsieh, Y. - J. Polym. Sci.: Part B: Polymer Physics, 34, p.1451 (1996).

41. Barkakaty, B. C. - J. Appl. Polym. Sci., 20, p.2921 (1976).

42. Hermans, P. H. \& Weidinger, A. - J. Polym. Sci., 4, p.135 (1949).

43. Atalla, R. H. - "The Structure of Cellulose" in Anais doMat. Res. Soc. Symp. Proc., 197, p.89 (1990).

44. Rowell, R. M.; Han, J. S. \& Rowell, J. S. - "Natural Polymers and Agrofibers Based Composites", Frollini, E.; Leão, A.; Mattoso, L. H. C. (eds), IQSC/Embrapa Instrumentação Agropecuária/UNESP, São Carlos (2000).

45. Chand, N.; Verma, S. \& Khazanchi, A. C. - J. Mater. Sci. Lett., 8, p.1307 (1989).

46. Bisanda, E. T. N. \& Ansell, M. P. - J. Mater. Sci., 27, p.1690 (1992).
47. Bisanda, E. T. N. - Ph.D. Thesis, University of Bath, UK (1991).

48. Lock, G. W. - "Sisal, Twenty-five Years Sisal Research", Longmans, Tanganyka (1962).

49. Chand, N. \& Hashmi, S. A. R. - J. Mat. Sci., 28, p.6724 (1993).

50. Greenberg, A. R.; Mehling, A.; Lee, M. \& Bock, J. H. - J. Mater. Sci., 24, p.2549 (1989).

51. Reddy, N. \& Yang, Y. - Polymer, 46, p.5494 (2005).

52. Keckes, J.; Burgert, I.; Frühmann, K.; Müller, M.; Kölln, K.; Hamilton, M.; Burghammer, M.;Roth, S. V.; Stanzl-Tschegg, S. \& Fratzl, P. - Nat. Mater., 2, p.810 (2003).

53. Fairbridge, C. \& Ross, R. A. - J. Appl. Polym. Sci., 22, p.497 (1978).

54. Bhaduri, S. K.; Mathew, M. D.; Day, A. \& Pandey, S. N. - Cell. Chem. Technol., 28, p.391 (1994).

55. Shafizadeh F. \& Bradbury, G. W. - J. Appl. Polym. Sci., 23, p.1431 (1979).

56. Chand, N.; Sood, S.; Singh, D. K. \& Rohatgi, P. K. - J. Therm. Anal. 32, p.595 (1987).

57. Martins, M. A.; Kiyohara, P. K. \& Joekes, I. - J. Appl. Polym. Sci., 94, p.2333 (2004).

Enviado: 20/06/08

Reenviado: $15 / 09 / 08$

Aceito: 20/09/08 